\title{
Estudio de la distribución de las especies frente al cambio climátíco
}

\section{J. Bravo Cadena', G. Sánchez Rojas ${ }^{1}$, \& S.M. Gelviz- Gelvez ${ }^{2}$}

Laboratorio de Biología de la Conservación ${ }^{\mathrm{I}}$ y Laboratorio de Comunidades ${ }^{2}$ Centro de Investigaciones Biológicas, Universidad Autónoma del Estado de Hidalgo Km 4.5 de la Carretera Pachuca Tulancingo Col. Taxistas 42 i 84 Mineral de la Reforma, Hidalgo Apartado postal 69-i Pachuca Hidalgo 4200 I email:gsanchez@Uaeh.edu.mX

\section{ABSTRACT}

Due to global warming in the last hundred years, there have been different approaches to assess their impact on biodiversity. Within these approaches, one that is frequently used is to evaluate changes in species distribution. This assessment uses long-term direct observations, the fossil record or spatially explicit models considering the climatic conditions of past, present and future. These models can be evaluated by direct observation or through the past with the fossil record. The results show that species can expand, maintain or reduce their distribution depending on the characteristics of the species. On the other hand, the information generated from these studies could help to identify the most vulnerable species to climate change, or those who can benefit, being useful to propose mitigation measures or conservation strategies.

Keywords: Climate Change, distribution, research lines.

\section{CAMBIO CLIMÁTICO}

Para entender el cambio climático primero es importante entender qué es el clima, este puede considerarse como la descripción estadística del tiempo (condiciones climáticas a corto plazo), expresada con los valores medios y su variabilidad en un periodo de tiempo determinado, que normalmente es de 30 años (IPCC 2007).

El Panel Intergubernamental de Cambio Climático (IPCC http://www.ipcc.ch/) define al cambio climático como "un cambio de clima atribuido directa o indirectamente a la actividad humana que altera la composición de la atmósfera mundial y que se suma a la variabilidad natural del clima observada durante períodos de tiempo comparables" (IPCC 2002). A lo largo de la historia de la tierra, el clima ha tenido grandes fluctuaciones, las cuales están relacionadas con los diferentes controles y factores del clima. Cuando se presentan estas fluctuaciones se registran cambios en la biodiversidad del planeta (TREJO, 1983). De hecho, estos cambios climáticos 
se presentan acompańando a las extinciones masivas que han ocurrido en el pasado (Fig. 1).

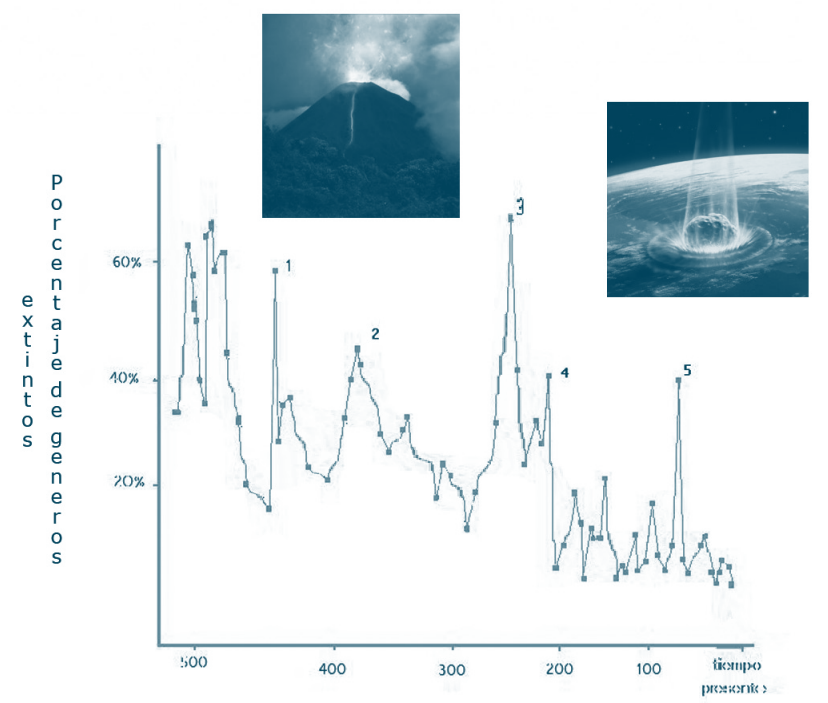

Milones de años antes del presente

Figura 1: Representación de los cambio de la diversidad biológica, a lo largo de los últimos quinientos cincuenta millones de años, donde se indica los cinco eventos previos de extinción masiva que se pueden registra mediante el estudio de los fósiles marinos, en todos los casos se han presentado grandes cambios en el clima que aceleran el procesos de extinción.

Es de especial interés el efecto que tenga sobre a la biodiversidad, el calentamiento global actual (que puede llevar, de continuar y no revertir las tendencias, a un cambio climático global), el cual se observa por el patrón que la temperatura ha mantenido desde el periodo 1906-2005 donde la temperatura global ha aumentado aproximadamente $0.74^{\circ} \mathrm{C}$ y en el caso de los polos el incremento es de casi el doble, lo cual puede estar relacionado con el aumento en las concentraciones de gases de efecto invernadero producidos por acciones humanas (IPCC 2007). Predicciones climatológicas IPCC, indican que en los siguientes cien años podría incrementarse la temperatura entre 1.1 y $6.4^{\circ} \mathrm{C}$ (IPCC 2007). De ahí que a esta época se le haya dado por nombrarla como el Antropoceno, donde este término ha sido empleado para describir el actual período en la historia terrestre iniciado desde que las actividades humanas han tenido un impacto global significativo sobre los ecosistemas terrestres. Este término lo acuño el premio Nobel de Química Paul Crutzen, quien considera que esta influencia del comportamiento humano sobre la Tierra en las recientes centurias es tan significativo que debería de considerarse como nueva era geológica (CRUTZEN \& STOERMER, 2000).

\section{EFECTO DEL CAMBIO CLIMÁTICO EN LA BIODIVERSIDAD}

Desde el punto de vista de la biodiversidad se ha documentado que el cambio climático afecta directa e indirectamente a individuos, poblaciones y especies, así como a los ecosistemas en su composición y función (PARMESAN, 2006, IPCC 2007, JANETOS et al., 2008). Este cambio puede generar pérdida del hábitat, de componentes del ecosistema y de interacciones intra- e interespecífica, así como aumento en la distribución de especies invasoras, modificaciones en los patrones de la migración de los organismos, en el tamaño y distribución de las poblaciones, entre otros aspectos (PETERSON et al., 2002, CRICK 2004, LEMOINE et al., 2007, BALLESTEROSBARRERA, 2008, HUANG \& GEIGER, 2008). Es decir, genera cambios dentro de la diversidad biológica tanto en su composición, estructura y función a diferentes escalas temporales y espaciales.

A nivel de las poblaciones, estas respuestas varían dependiendo de las especies y su capacidad de respuesta a los cambios, es decir, hay especies que tienen gran amplitud de su nicho (generalistas) que se pueden ver beneficiadas por las modificaciones en el clima por lo que podrían responder aumentando sus poblaciones e incluso aumentado su área de distribución geográfica. Sin embargo, existen otras especies con una reducida amplitud de su nicho (especialistas) que potencialmente se verán afectadas más drásticamente por los cambios, es decir tienen una mayor sensibilidad al efecto del cambio climático, estas especies están agrupadas en aquellas con: a) dependencia de un hábitat y/o un microhábitat especializado; b) reducida tolerancia o umbrales ambientales muy estrechos que son susceptibles de ser sobrepasados en cualquiera de las etapas del ciclo vital; c) dependencia de un detonante o señal ambiental específica que es susceptible de sufrir una perturbación; d) dependencia de interacciones interespecíficas susceptibles de sufrir perturbaciones; y limitada capacidad de dispersión o de colonización de zonas nuevas o más favorables (VIÉ et al., 2009). 
Se ha observado que entre el $70-80 \%$ de las aves, anfibios y corales que se encuentra clasificados como amenazados por la Unión Internacional para la Conservación de la Naturaleza (IUCN por sus siglas en inglés) son "sensibles al cambio climático" (VIÉ et al., 2009). En cuanto a las plantas se ha documentado la respuesta de algunas especies a diferentes concentraciones de $\mathrm{CO} 2$, encontrando que se presenta una alteración en la eficiencia fotosintética, en la fenología, tasas de crecimiento, así como en las comunidades lo que conlleva a una alteración en la estructura, dinámica y funcionamiento de los ecosistemas (MOONEY et al., 2001; REEKIE \& BAZZAZ, 1992).

La distribución de las especies entonces puede verse afectada debido a que están asociadas a un rango térmico de humedad y radiación que influye en la fenología y fisiología (VELÁZQUEZ, 2002) y estos rangos están asociados a ciertas ubicaciones geográficas en el planeta (es decir a su distribución geográfica, HUGHES, 2000). Ninguna especie, por generalista que sea, presenta poblaciones en todos los lugares, siempre encontraremos a las especies en un conjunto espacialmente discontinuo de fragmentos de hábitat adecuado inmersos en fragmentos de hábitat inadecuado a las especies (Fig. 2), lo que demuestra que tienen límites de tolerancia a factores ambientales (MACARTHUR, 1972; BEGON et al., 2006). Consecuentemente el cambio climático afectar la distribución de las especies (Hunthey, 1995; Parmesan \& Yohe, 2003) y de esta manera a la biodiversidad (LEEMANS \& HALPIN, 1992).

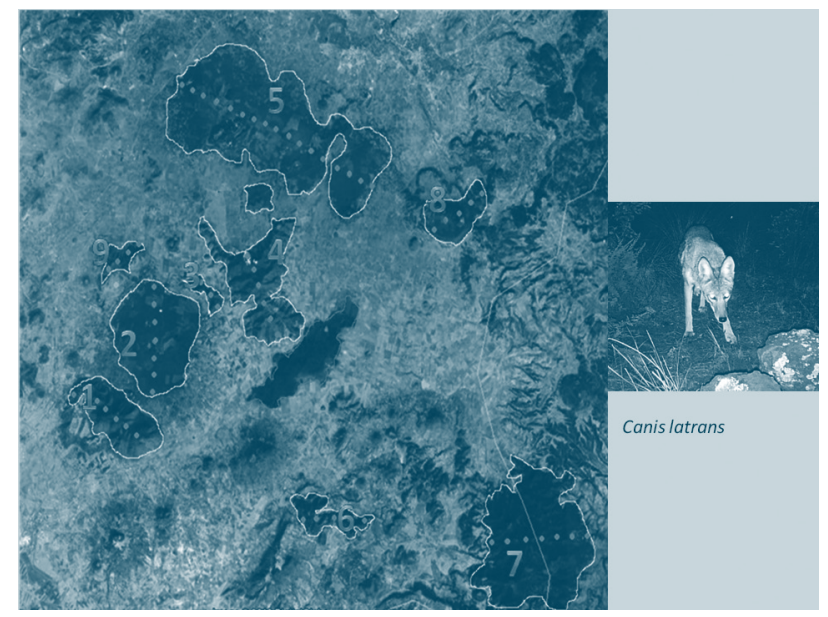

Figura 2
Figura 2: Ninguna especie tiene un hábitat adecuado continuo, generalmente estos se encuentran discontinuos y rodeados de hábitat inadecuado para los individuos de una especies, aquí se muestran algunos fragmentos de vegetación remanente que utiliza el coyote (Canis latrans), en la región de Apan Hidalgo. (Fotos cortesía de Alejandro García Becerra, y del Proyecto 92122 CONACYT-FOMIX-HIDALGO)

\section{EFECTO EN LA DISTRIBUCIÓN DE LAS ESPECIES}

Considerando sólo la línea de investigación de cómo afecta el cambio climático a la distribución de las especies se han generado un gran número de trabajos reportando observaciones directas de investigaciones de largo plazo, registrando los cambios en la distribución considerando los registros fósiles o generando modelos de distribución potencial hacia el pasado o proyecciones hacia el futuro.

Tomando en cuenta las observaciones directas o seguimiento a largo plazo de las poblaciones, uno de los ejemplos más documentados es la modificación de la distribución del oso polar a consecuencia del derretimiento del hielo marino (DEROCHER, 2005); la reducción de la distribución de los pingüinos en la Antártida (FORCADA, 2007); la reducción de la distribución de algunas especies de anfibios Neotropicales (Lips el al., 2005); por otro lado también se han observado especies que amplían su distribución como las especies invasoras, por ejemplo algunas especies de pastos (SMITH, 1994).

Los estudios que consideran los registros fósiles, los cuales utilizan diferentes proxies (por ejemplo polen, diatomeas, etc.) también están ampliamente desarrollados. Sólo por mencionar algunos, en México se han realizado estudios del cambio climático en Yucatán durante la pequeña edad de hielo, donde se muestra que no están presentes algunas especies de plantas durante ese periodo, la temperatura del mar se enfrió y el interior de la península se volvió más seca (HODELL et al., 2005), también se han desarrollado diferentes estudios en paleólagos, en el norte y centro del país y muestran que en los últimos 1500 años ha existido una marcada variación en el clima y fuertes presiones antropogenicas, lo 
cual determina la presencia o ausencia de especies en los sitios de estudio (CABALLERO et al., 2003 y METCALFE \& DAVIES, 2007).

Por último, una de las líneas de investigación más desarrolladas son los modelos de distribución espacialmente explícitos, considerando las condiciones climáticas actuales y los modelos climáticos del pasado y del futuro. Estos modelos permiten estimar la distribución potencial, para el tiempo en que el clima ha sido modelado. La predicción de la distribución de las especies con base en variables ambientales se fundamenta con la teoría de nicho formalizada por HUNTCHINSON (1957) y las predicciones que se hacen sobre la distribución de las especies bajo estos escenarios son llamados modelos de nicho ecológico (LAWLEY et al., 2006). Estos modelos al permitirnos modelar y por tanto presentar hipótesis de la distribución geográfica de las especies, es de enorme utilidad para abordar diferentes preguntas biológicas (GUISAN \& THUILLER, 2005; VÁZQUEZ, 2005).

Por mencionar algunas de las investigaciones que modelan la distribución hacia el pasado, se encuentran las proyecciones hacia el Pleistoceno, para explicar la diversidad genética de Melanoplus marshalli en Colorado y Utah (KNOWLES 2007) y para modelar la distribución 23 especies de mamíferos (MARTÍNEZ-MEYER et al., 2004). Se ha modelado también la distribución de 20 vertebrados terrestres identificando refugios pleistocenicos, además de comprar los resultados generados con técnicas genéticas y proyecciones filogenéticas, en donde se muestra que los resultados obtenidos con los modelos están correlacionados con los obtenidos con las otras técnicas (WALTARI et al., 2007).

Sin embargo, pocas veces se utilizan datos independientes para evaluar los modelos de distribución en el pasado, por lo que se pueden generar diferentes errores en la interpretación de los datos (NOGUÉSBRAVO, 2009). A pesar de esto, los modelos de distribución proyectados al pasado tienen mayor posibilidad para ser evaluados, ya que se puede tener la evidencia de la distribución con el registro fósil. Algunos trabajos están enfocados hacia la hipótesis del conservadurismo de nicho (basada en que las especies, más que adaptarse a los cambios climáticos, se mueven a colonizar nuevos hábitats que tengas las características de su nicho (MARTÍNEZ-MEYER $\&$ PETERSON, 2006).

Considerando los modelos de distribución potencial de las especies con diferentes escenarios de cambio climático hacia el futuro. Estos se han desarrollado a diferentes escalas y utilizando diferentes set de datos. Por mencionar algunos, se ha modelado la distribución potencial de cactáceas en la Reserva de la biosfera Tehuacán-Cuicatlán bajo escenarios de cambio climático, en donde se muestra que para las especies endémicas de cactáceas puede reducirse la distribución en más del 50\% (TÉLLEZ-VALDÉS \& VILA-ARANDA, 2003); también se han generado los modelos de distribución de dos especies de salamandras en el centro de México bajo escenarios de cambio climático, en donde observan que incluso las especies ampliamente distribuidas, pueden verse amenazadas por el cambio climático en los próximos 50 años (PARRA-OLEA et al., 2005). Se ha modelado la distribución pasada, presente y futura de diversas especies en el Desierto Chihuahuense, mostrando en las proyecciones futuras, que la fragmentación del hábitat aunado al calentamiento global pondrá en riesgo de extinción a las especies de distribución restringida, poco vágiles y especialistas (BALLESTEROS-BARRERA, 2008).

En otras partes del mundo se han realizado un gran número de trabajos. Por mencionar algunos, se ha estudiado la eficiencia de las áreas para la conservación de aves (AICAs) en África, bajo la óptica del cambio climático, y los resultados muestran que la mayoría de las especies pueden reducir drásticamente su área de distribución, muchas AICAs pierden especies y en pocas hay reemplazamiento (COETZEE $e t$ al., 2009); se han realizado modelos de distribución de especies alpinas, considerando proyecciones de cambio climático en las zonas montañosas, que muestran que los modelos con escalas finas pueden reflejar mejor los requerimientos climáticos en las zonas más altas (RANDIN, 2009).

Además se han modelado 85 especies de plantas 
subalpinas a una resolución de 20X20 m y predicen que por arriba del $50-60 \%$ de las especies estudiadas pueden extinguirse (DIRNBÖCK et al., 2003); de las plantas montańosas de Europa el 60\% pueden ser susceptibles al cambio climático ya que en los modelos muestran una reducción de más de 10\% de su área de presencia (THUILLER et al., 2005)

\section{CONCLUSIONES}

Existen varias alternativas para investigar la respuesta de la distribución de las especies al cambio climático, sin duda las más precisas son las observaciones directas o investigaciones a largo plazo. Cuando se amplía la escala de tiempo de interés, las investigaciones más precisas pueden ser los estudios paleontológicos. Por otro lado los modelos de distribución potencial son una herramienta muy importante en el estudio el cambio climático y la biodiversidad ya que pueden generar la materia prima para identificar las especies más vulnerables al cambio climático, así como aquellas especies que pueden ser beneficiadas, esta información es utilizada para generar diversas estrategias de conservación y/o manejo a diferentes escalas.

\section{AGRADECIMIENTOS}

Este trabajo conto con el apoyo del proyecto FOMIX-HIDALGO 98122 "Efecto del cambio Climático en la Distribución de las Diversidad en el Estado de Hidalgo"

\section{BIBLIOGRAFÍA}

BALLESTEROS-BARRERA, C. 2008. Efecto del cambio climático global en la distribución de especies del Desierto Chihuahuense. Tesis de Doctorado. Universidad Nacional Autónoma de México. Facultad de Ciencias. México D.F.

BEGON, M., C. TOWNSEND \& J. HARPER. 2006. Ecology from individuals to ecosystems. Blackwell Publishing. U.S.A. 752 pp.
CABALLERO, M., G. VILACLARA., A. RODRIQUEZ \& D. JUAREZ. 2003. Short term climatic change in lake sediments from Lake Alchichica, Cuenca Oriental, México. Geof Int. 42:529-537.

COETZEE, B. W. T., M. P. ROBERTSON., B. F. N. ERASMUS., B. J. VAN-RENSBURG \& W. THUILLER. 2009. Ensemble models predict Important Bird Areas in southern Africa will become less effective for conserving endemic birds under climate change. Glob Ecol and Biogeogr. 1:1-10.

CRICK, H. Q. 2004. The impact of climate change on birds. Ibis. 146:48-56.

CRUTZEN, P. J \& E. F. STOERMER. 2000. The Anthropocene. Glob Change News 41:17-18.

DEROCHER, A. 2005. Population ecology of polar bears at Svalbard, Norway. Popul Ecol. 47:267-275.

DIRNBÖCK, T., S. DULLINGER \& G. GRABHERR. 2003. A regional impact assessment of climate and landuse change on alpine vegetation. J Biogeogr. 30:401-417.

FORCADA, J. 2007. Cambio climático y sus repercusiones en la megafauna de la Antártica. En: Duarte (Eds) Impactos del calentamiento global sobre los ecosistemas polares. Disponible en: http://dialnet.unirioja.es

GUISAN, A \& W. THUILLER. 2005. Predicting species distribution: offering more than simple habitat models. Ecol Lett. 8:993-991.

HODELL, D., M. BRENNER., J. CURTIS., R. MEDINAGONZALEZ., E. ILDEFONSO-CHAN., A. ALBORNAZ-PAT \& T. GUILDERSON. 2005. Climate change on the Yucatan peninsula during the little ice age. Quat Res 63:109-121.

HUANG, C \& E. GEIGER. 2008. Climate anomalies provide opportunities for large-scale mapping of non-native plant abundance in desert grasslands. Divers Distrib. 14:875-884.

HUGHES, L. 2000. Biological consequences of global warming: is the signal already apparent?. Trends Ecol. Evol. 15: 56-61.

HUNTHEY, B. 1995. Modelling present and potential futures ranges of some Eutopean higher plats using clite response surface. J Biogeogr. 22:967-101.

HUNTCHINSON, G. E. 1957. Concluding remarks. Cold Spring Harbor Simposia on Quantitative Biology 22:415-427. 
IPCC，2002. Cambio Climático y Biodiversidad. Unidad de Apoyo Técnico del Grupo de Trabajo II del IPCC Gitay, H., Suárez, A. Watson R. y Dokken D. (Editores). Unidad de Apoyo Técnico del Grupo de Trabajo II del IPCC. IPCC, Ginebra, Suiza.

IPCC, 2007. Cambio climático: Informe de síntesis. Contribución de los Grupos de trabajo I, II y III al Cuarto Informe de evaluación del Grupo Intergubernamental de Expertos sobre el Cambio Climático [Equipo de redacción principal: Pachauri, R.K. y Reisinger, A. (directores de la publicación)]. IPCC, Ginebra, Suiza.

JANETOS, A., L. HANSEN., D. INOUYE., B. P. KELLY., L. MEYERSON., B. PETERSON \& R. SHAW. 2008. Biodiversity. En: Walsh M. (Edt)The effects of climate change on agriculture, land resources, water resources, and biodiversity. A Report by the U.S. Climate Change Science Program and the Subcommittee on Global Change Research. Washington. Pp. 362.

KNOWLES, L. L., B. C. CARSTENS \& M. L. KEAT 2007. Coupling genetic and ecological-niche models to examine how past population distributions contribute to divergence. Curr Biol. 17:940-946.

LAWLEY, J., D. WHITE, R. NEILSON \& A. BLAUSTEIN. 2006. Predicting climate-induced range shifts: model differences and model reliability. Glob Change Biol. 12:1568-1584.

LEEMANS, R \& P. HAPIN. 1992. Global change and biodiversity. En: Chapman \& hall (eds.) biodiversity 1992: status of the earth's living resources. London.

LEMOINE, N., B. BAUER., M. PEINTINGER \& K. BÖHNING-GAESE. 2007. Effects of Climate and Land-Use Change on Species Abundance in a Central European Bird Community. Conserv. Biol. 21:495-503.

LIPS K. R. , P. A. Burrowes , J. R. Mendelson, Parra-Olea G. 2005. Amphibian Population Declines in Latin America: A Synthesis. Biotropica 37:222-226.

MARTÍNEZ-MEYER, E., A. T. PETERSON \& W. W. HARGROVE. 2004. Ecological niches as stable distributional constraints on mammal species, with implications for Pleistocene extinctions and climate change projections for biodiversity. Glob Ecol Biogeogr. 13:305-314.

MARTÍNEZ-MEYER, E \& A. T. PETERSON. 2006. Conservatism of ecological niche characteristics in North American plant species over the Pleistocene-to-Recent transition. J Biogeogr. 33:1779-1789.
MACARTHUR, R. 1972. Geographical ecology: patterns in the distribution of species. Harper and Row, New York. 269 pp.

METCALFE, S. 2007. Deciphering recent climate change in central Mexican lake records. Clim Change. 83:169-186.

MOONEY, H. A., B. G. DRAKE., R. J. LUXMOORE., W. C. OECHEL \& L. F. PITELKA. 1991. Predicting Ecosystem Responses to Elevated CO2 Concentrations: What has been Learned from Laboratory Experiments on Plant Physiology and Field Observation? Bioscience. 41: 96-104.

NOGUÉS-BRAVO, D. 2009. Predicting the past distribution of species climatic niches. Glob Ecol Biogeogr. 18: $521-531$

PARRA-OLEA, G., E. MARTÍNEZ-MEYER, \& G. PÉREZPONCE DE LEON. 2005. Forecasting climate change effects on salamander distribution in central Mexico highlands. Biotropica 37: 202-208.

PARMESAN, C. 2006. Ecological and evolutionary responses to recent climate change. Ann Rev Ecol Evol Syst. 37:637-669.

PARMESAN, C \& G. YOHE. 2003. A global coherent fingerprint of climate change impacts across natural systems. Nature. 421:37-42.

PETERSON, A. T., M. A. ORTEGA-HUERTA., J. BARTLEY., J. SÁNCHEZ-CORDERO., J. SOBERÓN., R. H. BUDDEMEIER \& D. R. STOCKWELL. 2002. Future projections form Mexican faunas under global climate change scenarios. Nature 416: 626-629

RANDIN, C. F., R. ENGLER., S. NORMAND., M. N. ZAPPA., E. ZIMMERMANN., P. B. PEARMAN., P. VITTOZ., W. THUILLER \& A. GUISAN. 2009. Climate change and plant distribution: local models predict high-elevation persistence. Glob Change Biol. 15:1557-1569.

REEKIE, E. G \& F. A. BAZZAZ. 1992. Phenology and Growth in Four Annual Species Grown in Ambient and Elevated CO2. Can J Bot. 69: 2475-2481.

SMITH, D. R. 1994. Change and variability in climate and ecosystem decline in Aral Sea basin deltas. Post-Soviet Geography 35:142-165.

TÉLLEZ-VALDÉS, O \& P. VILA-ARANDA. 2003. Protected areas and climate change: a case study of the cacti in the Tehuacán-Cuicatlán Biosphere Reserve, Mexico. Conserv Biol. 17:846-853. 
THUILLER, W., S. LAVOREL., M. B. ARAUJO., M. T. SYKE., I. C. PRENTICE. 2005. Climate change threats to plant diversity in Europe. Proc Natl Acad Sci USA. 102: 8245-8250.

TREJO H. 1983. Paleobiología y Taxonomía de Algunos Fósiles Mesozoicos de México. Boletín de la Sociedad Geológica Mexicana. 1-22.

VÁZQUEZ, D. 2005. Reconsiderando el nicho hunchinsoniano. Ecol. Austral. 15:149-158.
VELÁZQUEZ, J. 2002. Agrofenoclimatología. México, Universidad Autónoma del Estado de México. 338 p.

VIÉ, J. C., C. HILTON-TAYLOR \& S. N. STUART. 2009. Wildlife in a Changing World - An Analysis of the 2008 IUCN Red List of Threatened Species. Gland, Switzerland: IUCN.

WALTARI E., R. HIJMANS., A. PETERSON., A. NYÁRI., S. PERKINS., R. GURALNICK. Locating Pleistocene Refugia: Comparing Phylogeographic and Ecological Niche Model Predictions. PLoS ONE. 2007;2:e563. doi: 10.1371/journal.pone.0000563. 